\title{
Spectral dependence of diffuse light dynamics in ultracold atomic ${ }^{85} \mathrm{Rb}$
}

\author{
S. Balik, R. Olave, C.I. Sukenik, and M.D. Havey \\ Department of Physics, Old Dominion University, Norfolk, VA 23529 \\ V.M. Datsuk, D.V. Kupriyanov and I.M. Sokolov \\ Department of Theoretical Physics, State Polytechnic University, 195251, St.-Petersburg, Russia
}

\begin{abstract}
We report a combined experimental and theoretical simulation of multiply scattered light dynamics in an ultracold gas of ${ }^{85} \mathrm{Rb}$ atoms. Measurements of the spectral dependence of the time-decay of the scattered light intensity, following pulsed excitation with near resonance radiation, reveals that the decay for long times is nearly exponential, with a decay constant that is largely independent of detuning from resonance. Monte Carlo simulations of the multiple scattering process show that, for large detunings, near resonance scattering of Fourier components of the excitation pulse plays a significant role in the effect. This interpretation is supported by the observations, and successful modelling, of beating between Rayleigh scattered light at the excitation carrier frequency with the Fourier components of the excitation pulse that overlap significantly with the atomic resonance.
\end{abstract}

PACS numbers:

\section{INTRODUCTION}

Multiple scattering of near-resonance radiation is well known to have significant effects on the density distribution of trapped ultracold atoms [1], and on the collective dynamics of the trapped ensemble itself [2]. However, in spite of the enormous number of applications and studies of atoms confined in a MOT, there have been relatively few direct studies of the dynamics of the multiply scattered light itself. The limited number of studies include measurements of radiation trapping [3] of resonance radiation in an ultracold gas of atomic Cs by Fioretti, et al. [4] and studies of the long-time dynamics of light scattered in the vicinity of the atomic ${ }^{85} \mathrm{Rb}$ $F=3 \rightarrow F^{\prime}=4$ resonance transition [5, [6]; these measurements were also made in an ultracold gas, for which there is limited frequency redistribution of the scattered light. Recent measurements by Balik, et al. [7] examined the influence of quantum interference on the dynamics of the atomic alignment produced in the vicinity of the $F=3 \rightarrow F^{\prime}=4$ hyperfine transition associated with the atomic ${ }^{85} \mathrm{Rb} \mathrm{D} 2$ resonance transition.

Multiple light scattering is also important in several specific areas of current research interest [8 $[12]$. These areas are focussed on the role of interferences in multiple wave scattering in a wide variety of condensed, liquid, or gaseous samples. Such interferences are readily observable in many condensed systems and under a broad range of circumstances. For warm atomic gases, the interferences are normally not observable because of frequency redistribution caused by the thermal motion of the constituent atoms or molecules. However, in ultracold atomic gases [13 16], where the thermal motion of the atoms is very small, the interferences become readily observable through the coherent backscattering effect [17 19]. Research to date has recently been reviewed by Havey, et al. [20] and by Kupriyanov, et al. [21]. In relatively low density gases, where the mean free path for light scattering is much greater than the mean separation between the atoms, the process can be considered to be due to a sequence of separate scattering and propagation events. All studies to date have been done in the so-called weak localization regime, defined by the Ioffe-Regel condition as occurring when $\mathrm{k} l>>1$. Here $k$ is the wave vector of the light, while $l$ is the mean free path for light propagation in the medium. The coherent backscattering enhancement is due to distributions of chains of such sequences with a corresponding distribution of scattering orders. Scattering along reciprocal paths then leads to the interferometric enhancement of light scattered in the backscattering direction; in this direction the relative geometrical phase of the reciprocal paths is preserved, irrespective of the location of the atoms comprising the chains. When the density of atoms is increased to the point where the mean free path for light scattering is on the order of the atomic separation, then recurrent scattering [8] becomes important, and a transition from propagating light modes to localized ones becomes possible. The boundary near which this is expected to become important is defined by the Ioffe-Regel condition as $\mathrm{k} l \sim$ 1. Localization by disorder (in this case disorder due to the random distribution of atoms in the sample) is the optical analog of Anderson localization 22] of electrons. One driving motivation for studies of diffusive light propagation is to understand the purely diffusive part of the light propagation, and how the contrast with a transition to localized modes might be observed. Light localization has been reported for condensed samples in the optical [10] and in the microwave regimes [11], but not in an atomic gas.

A second area of considerable interest is coherent manipulation of light propagation in ultracold atomic gases 23]. This is accomplished by using effects associated broadly with coherent population trapping [24 26], and specifically under conditions associated with electromagnetically induced transparency (EIT) [27 30]. One re- 
markable demonstration of this was the seminal experiment by Hau, et al. [31], in which reduction of the speed of a coherent beam of resonance radiation to $17 \mathrm{~m} / \mathrm{s}$ in ultracold atomic sodium was observed. In this experiment, the group velocity of a weak probe beam was manipulated by a second control beam, where the two light sources were tuned to a two-photon Raman resonance between $\mathrm{Na}$ ground state hyperfine levels. Other experiments have used EIT to demonstrate nonlinear optical effects including four-wave mixing [32], optical information storage [33], remarkable recent experiments demonstrating generation and control of single photons 34 [36], and general studies of EIT phenomenology in ladder and lambda type configurations [37]. All such studies have been concerned with single or multiple coherent beams either applied or generated by nonlinear processes in the samples. However, as demonstrated by the coherent backscattering effect, phase can also be preserved in multiple scattering of light in an ultracold atomic gas [38]. This leads to the possibility that the CBS effect, in the weak localization regime, can be modified by the presence of a second control field in one of the customary EIT configurations. Recent theoretical results have in fact demonstrated that profound effects on the CBS intensity profile, spectral variation, and the temporal pulse shape of multiply scattered light beams are greatly modified by the presence of a control beam [39]. A physical way to envision these effects is to realize that the CBS effect reflects the optical transport properties of a weak probe beam, and the presence of an additional control field modifies these transport properties. This modification of the diffuse light propagation in an EIT configuration is a newly discovered effect, and much exploration of the experimental and theoretical landscape remains to be done.

\section{EXPERIMENTAL APPROACH}

The experimental details of our approach have been described elsewhere [7], and will be only briefly sketched here. The basic experimental scheme is portrayed in Fig. 1 , where it is shown that physical sample is an ultracold gas of atomic ${ }^{85} \mathrm{Rb}$ prepared in a magneto optical trap. The trap operates on the $F=3 \rightarrow F^{\prime}=4$ hyperfine transition. The trap [16] produces a nearly Gaussian cloud of $\sim 10^{8}$ ultracold rubidium atoms at a temperature $\sim 100$ $\mu \mathrm{K}$. The peak density is $\sim 3 \times 10^{10} \mathrm{~cm}^{-3}$. Fluorescence imaging is used to estimate the Gaussian radius of the sample to be $r_{0} \sim 1 \mathrm{~mm}$. The peak optical depth, as measured by absorption measurements of light transmitted through the central portion of the atomic sample, is $b_{0}=$ 8(1). For a Gaussian atom distribution in the trap, the weak-field optical depth, on resonance and through the center of the trap, is given by $b_{0}=\sqrt{2 \pi} n_{0} \sigma_{0} r_{0}$, where $n_{0}$ is the peak trap density and $\sigma_{0}$ is the on-resonance cross-section. The single resonance scattering cross section $\sigma$ varies with probe frequency, $b=b_{0}\left[1+(2 \Delta / \gamma)^{2}\right]^{-1}$,

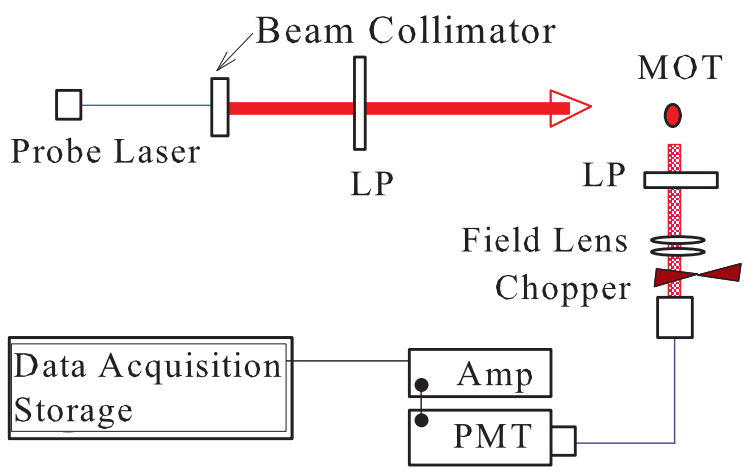

FIG. 1: Schematic diagram of the experimental arrangement. Shown in the figure is the probe laser, magneto optic trap (MOT), linear polarizers (LP), and a photomultiplier tube (PMT) used for detection of the fluorescence signals.

where $\Delta=\omega_{L}-\omega_{0}$, and $\omega_{L}$ is the probe frequency, $\omega_{0}$ is the $F=3 \rightarrow F^{\prime}=4$ hyperfine transition frequency, and $\gamma$ is the natural width. A continuous wave, low intensity diode laser serves as probe of the optical transmission of the MOT and also as the excitation source for the time resolved experiments reported here. The laser, which has a bandwidth $\sim 1 \mathrm{MHz}$, is tuned in a range of several $\gamma$ around this transition. The average light intensity for the probe is $\sim 1 \mu \mathrm{W} / \mathrm{cm}^{2}$. To produce a nearly Gaussian beam profile, the laser output is passed through a single-mode optical fiber and expanded to a $1 / e^{2}$ width $\sim 8 \mathrm{~mm}$. The probe laser intensity is modulated with an acousto optic modulator (AOM), which generates sharp rectangular pulses having an on time of $2 \mu s$ and an off time of $2 \mathrm{~ms}$. The $2 \mu \mathrm{s}$ excitation pulse is centered in a 90 $\mu s$ window during which fluorescence data are recorded. The MOT lasers are off during the data acquisition period. For the remaining nearly $2 \mathrm{~ms}$, the MOT lasers are turned back on to regenerate the ultracold atomic sample. An important component of the experiment is a synchronized mechanical chopper, which prevents intense fluorescence from the MOT region, present during the MOT build up period, from reaching the photomultiplier tube (PMT). The AOM-limited $20 \mathrm{~dB}$ response is estimated to be less than $\sim 60 \mathrm{~ns}$. The probe laser is linearly polarized in the vertical direction.

Fluorescence signals are detected in a direction orthogonal to the probe laser propagation and polarization directions. To minimize reduction in the measured light polarization, the scattered light is collected in a small effective solid angle of about $0.35 \mathrm{mrad}$, and refocussed to match the numerical aperture of a $400 \mu \mathrm{m}$ multimode fiber. A linear polarization analyzer is used to select the detected polarization channel, which we label as parallel $(\|)$ and perpendicular $(\perp)$. The fiber output is filtered by a $5 \mathrm{~nm}$ spectral width interference filter centered at $780 \mathrm{~nm}$, and passed to a GaAs-cathode photomultiplier tube (PMT). The PMT is operated in a photon counting 
mode, whereby the output is amplified and directed to a discriminator and multichannel scalar (MCS) (indicated in Fig. 1 as data acquisition and storage). The multichannel scaler time sorts and accumulates the data in 5 ns bins. Timing of the experiment, including the MOT and probe lasers, and triggering the start of the MCS sweep is controlled by a precision pulse generator slaved to a timing signal from the mechanical chopper.

\section{THEORETICAL APPROACH}

We provide here only an outline of our theoretical approach to description of the time evolution of light scattering in an ultracold atomic gas. Details of this treatment may be found in our earlier papers [7, 16, 20, 21, 39] The time dependence of the scattered pulse intensity can be extracted by using the following calculation steps. Basically the process is fully described by the scattering amplitude defined for each spectral component of the incoming and outgoing pulse. Thus, this scattering amplitude has to be established as a first step. Such an amplitude for the multiple scattering process developing in an opaque medium includes two important calculation ingredients, namely, the scattering tensor and the mesoscopic Green function. The description of these theoretical characteristics of the scattering problem for the relevant case of an ultracold atomic sample can be found in Ref.[21]]. Roughly speaking the amplitude can be constructed for any randomly chosen chain of atomic scatterers as a subsequent product of the respective scattering tensors responsible for the deflection of the light ray from its coherent forward propagation. The transformation of the field amplitude inside such a zigzag-path, but along the fragments of freely propagation, can be described in terms of the Green function formalism.

In the next step the partial contribution to the time profile of the outgoing light pulse is recovered via a reverse Fourier transform. This transform should be subsequently evaluated for each order of the multiple scattering. This part of the simulation procedure is the most difficult and needs an accumulation of the quite extended database for the large number of the contributing spectral components, see [39] for details. Finally the partial contribution to the instantaneous intensity of the light scattered by the selected chain is expressed by the squared time dependent partial amplitude. The crucial point of the final calculational step comes from the important physical requirement to the interference contribution associated with the scattering in backward direction. For this particular scattering channel the partial amplitudes responsible for the light propagation along two reciprocal scattering path can interfere and the interference survives the configuration averaging. At the final step the normal "ladder"-type and, in the case of the backscattering channel, the "crossed"-type amplitude products should be averaged with the Monte Carlo technique for a Gaussian-type distribution of the atomic scat- terers in the sample. The total outgoing pulse is finally expressed by the sum of such partial contributions, and the sum is typically converged for the orders of multiple scattering $\sim b_{0}$.

The described procedure of numerical simulation allows us to include in the numerical routine most of the practically important factors such that the hyperfine and Zeeman structure of the ground and excited states, the spatial inhomogeneity of atomic cloud and its shape variation. In addition, the dynamical modification of the scattering process caused by atomic motion can be traced via the time and spectral dependencies of the correlation function of the scattered light. Some interesting and important effects for the light scattering on spin-oriented atomic systems were predicted with such a detail numerical analysis, see 21].

\section{RESULTS AND DISCUSSION}

In an earlier paper we reported on the time dependence of the linear polarization degree of the multiply scattered light. In the measurements, the ultracold atoms are excited by a nearly rectangular $2 \mu$ s long pulse of linearly polarized resonance radiation tuned in the spectral vicinity of the $F=3 \rightarrow F^{\prime}=4 \rightarrow F=3$ transition. The measured intensities of linearly polarized scattered light in two orthogonal directions (along and perpendicular to the direction of the exciting light pulse polarization) may be quantified by defining a linear polarization degree as

$$
P_{L}=\frac{I_{\|}-I_{\perp}}{I_{\|}+I_{\perp}}
$$

In the formula, $I_{\|}$and $I_{\perp}$ represent the measured intensities in the lin $\|$ lin and lin $\perp$ lin channels. It was shown by Balik, et al. [7] that the linear polarization degree decays rapidly towards zero on a time scale of less than $100 \mathrm{~ns}$. In the present paper, we are concerned with the longer time dynamics, for which $I_{\|}$and $I_{\perp}$ very nearly equal. Our results are then presented solely in terms of $I_{\|}$.

The time dependence of the scattered light intensity $I_{\|}$, for several different detunings from the $F=3 \rightarrow F^{\prime}=4$ transition is shown in Fig. 2. There we see that, after a transient growth period of approximately $100 \mathrm{~ns}$, the fluorescence intensity reaches a steady state value. Upon shut off of the excitation pulse at a time $t=2000 \mathrm{~ns}$, the fluorescence decays to a small level on a time scale of several hundred ns. As the frequency of excitation is shifted away from the resonance transition, both the transient build up and decay become more rapid. This qualitatively makes sense, for the optical depth of the vapor is reduced with detuning from resonance, and the radiation thus undergoes fewer scattering events before emerging from the optically dense sample. As indicated by the solid lines in Fig. 2, the overall time dependence is well described by the results of Monte Carlo simulations. 


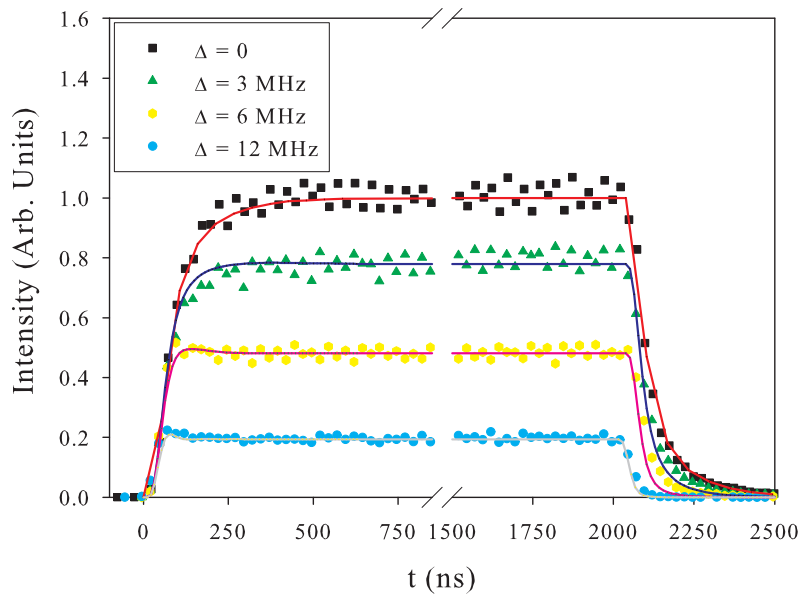

FIG. 2: Time-dependence of the scattered light intensity, in the $I_{\|}$polarization channel, for several different detunings from atomic resonance.

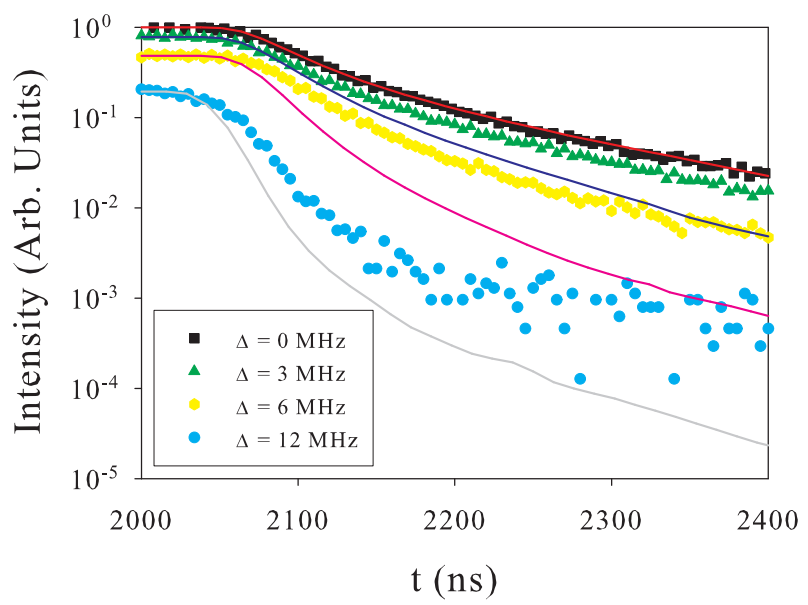

FIG. 3: Semilogarithmic graph of the time-dependence of the scattered light intensity, in the $I_{\|}$polarization channel, for several different detunings from atomic resonance.

The behavior of the intensity after the excitation pulse is turned off is seen more clearly in the semilogarithmic plots shown in Figure 3. There we see a surprising result: the long time decay is nearly exponential and has essentially the same decay rate, regardless of the detuning. We point out that, even though this data is for positive detunings of $\Delta=0,3,6$, and $12 \mathrm{MHz}$, a very similar effect is seen for negative detunings of the same magnitude. It is also evident that the Monte Carlo simulations, shown in this graph as solid lines, do not account for the effect just described. This suggests that additional physics must be included to better describe the physical circumstances of the experiment.

One possibility is that the finite bandwidth of about 1 $\mathrm{MHz}$ of the excitation laser results in resonance excitation with the wings of the laser spectral profile. Simula-

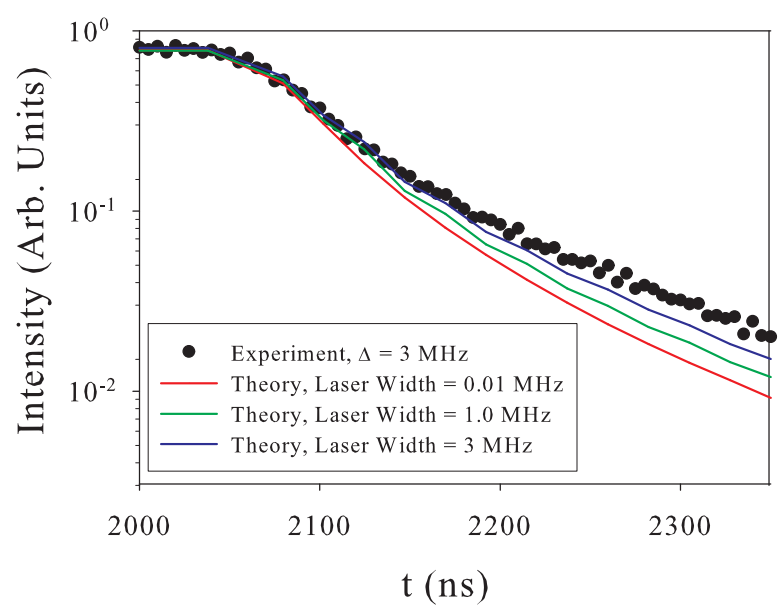

FIG. 4: Influence of the laser linewidth on the scattered light intensity, in the $I_{\|}$polarization channel, for a detuning $\Delta=$ $+3 \mathrm{MHz}$.

tions of this effect for laser widths of $0.01 \mathrm{MHz}, 1.0 \mathrm{MHz}$, and $3.0 \mathrm{MHz}$ are shown in Fig. 4. There it is seen that for small detunings $(\Delta=3 \mathrm{MHz}$ in this case), there is some influence, and the change goes in the proper direction in comparison with the experimental data. However, the bandwidth effect becomes increasingly negligible for larger detunings, and so cannot account for the results of Fig. 3.

The most promising possibility is that spectral components of the excitation pulse, generated by the relatively rapid turn on and turn off of the pulse, sufficiently overlap the atomic resonance transition to explain the effect. In Fig. 5 we compare the experimental data at a larger detuning of $\Delta=+12 \mathrm{MHz}$ with simulations for pulses having different turn-on and turn-off models and time constants. There it is seen, for example, that for an exponential turn off shape with a time constant on the order of a few ns, the data and simulations are in reasonable accord. As the Fourier components for a rectangular pulse drop off with frequency relatively slowly with offset from the carrier frequency, this is a suggestive interpretation of the observations. We point out that this interpretation is supported by the transient turn on behavior, as shown in Figure 6. There we see a sharp overshoot, which we interpret as beating between the Rayleigh scattered light and the Fourier components of the excitation pulse scattered according to the Lorentzian response of the atomic resonance. Although the experimental data is too noisy to extract the beat frequency, the simulations, on an expanded intensity scale, show that the beat note frequency is in fact given by the detuning from resonance.

Finally, we point out that Labeyrie, et al. [5, 6] have observed a similar effect in ${ }^{85} \mathrm{Rb}$ but in a somewhat different fluorescence geometry, sample geometry, and preparation. In these studies, the Gaussian radius of the MOT is $2-3 \mathrm{~mm}$, while the peak optical depth $b_{0}$ ranges from 


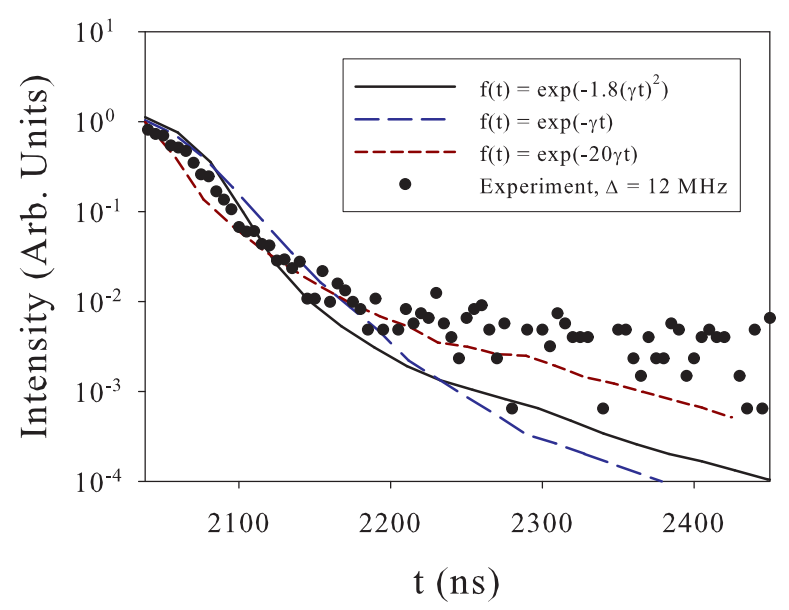

FIG. 5: Influence of the temporal shape of the excitation pulse on the long time behavior of the scattered light intensity in the $I_{\|}$polarization channel. $\Delta=+12 \mathrm{MHz}$.

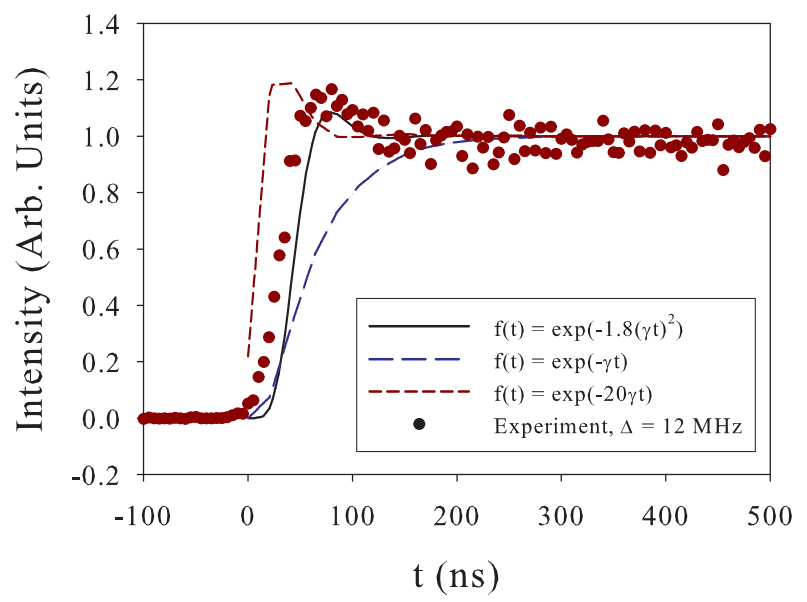

FIG. 6: Influence of the temporal shape of the excitation pulse on the short-time growth of the scattered light intensity in the $I_{\|}$polarization channel. $\Delta=+12 \mathrm{MHz}$.

2 to 40. In addition, optical excitation of the sample was done with a probe laser smaller than the sample size, in contrast to the experiments reported here. Finally, the angle of observation of the fluorescence was about $17^{\circ}$ from the forward direction, in comparison to our measurements where this angle is $\sim 90^{\circ}$. They interpret the long time constant as being due to redistribution of the multiply scattered light by the residual thermal motion of the atoms in the ultracold atomic cloud. Reported simulations are also in good agreement with their temperature dependent measurements of the decay rate at longer times. No data on the turn on portion of the fluorescence measurements were reported. In comparing the results of Labeyrie, et al. [5, 6] we see that the essential effect, that the longer time decay is nearly constant and independent of detuning, is the same in both experiments. However, the interpretation of the results is different. This could well be due to the quite different experimental arrangements and conditions for the two experiments, under which different physical mechanisms may be important. For the experiments reported here, it seems that the interpretation based on overlapping Fourier components provide a self consistant explanation for both the longer time decay and for the shorter time turn on transient. However, we should point out that initially warmer atoms also have a larger overlap with the Fourier components of the excitation pulse, and can contribute to the long time tail. This should have a prompt effect on the transient buildup and decay, rather than an time-accumulating effect such as frequency redistribution, due to thermal motion, in multiple scattering. Finally, the data reported in Fig. 5, for example, show not ideal agreement with our simulations, even accounting for the Fourier components of the excitation pulse. This residual difference may be due to thermal redistribution, which has not been taken into account in our simulations.

\section{CONCLUSIONS}

In conclusion, we report experimental and theoretical results on the long time decay of near resonance radiation from an ultracold gas of Rb atoms. The experimental results show two features suggesting that Fourier components of the excitation pulse play an important role in the observed constancy of the scattered light intensity at longer times following pulse shut off. Our simulations also show that realistic laser spectral widths can influence the observations for smaller detunings from resonance. Measurements simulations by other researchers, on the other hand, have shown that frequency redistribution due to residual thermal motion of atoms in the cold gas plays a significant role in interpreting off resonance light diffusion in an ultracold atomic sample.

Supported by the National Science Foundation (NSFPHY-0355024), by the Russian Foundation for Basic Research (RFBR-05-02-16172-a), and by the North Atlantic Treaty Organization (PST-CLG-978468).
[1] Harold J. Metcalf and Peter van der Straten, Laser Cooling and Trapping (Springer, New York, 1999).

[2] G. Labeyrie, Michaud, R. Kaiser, submitted to Phys.
Rev. Lett. (2005).

[3] T. Holstein, Phys. Rev. 72, 1212 (1947).

[4] A. Fioretti, A.F. Molisch, J.H. Muller, P. Verkerk, M. 
Allegrini, Opt. Comm. 149, 415 (1998).

[5] G. Labeyrie, E. Vaujour, C.A. Muller, D. Delande, C. Miniatura, D. Wilkowski, R. Kaiser, Phys. Rev. Lett. 91, 223904 (2003).

[6] G. Labeyrie, R. Kaiser, and D. Delande, Appl. Phys. B 81, 1001 (2005).

[7] S. Balik, R. G. Olave, C. I. Sukenik, and M. D. Havey, Phys. Rev. A 72, 051402(R) (2005).

[8] P. Sheng, Introduction to Wave Scattering, Localization, and Mesoscopic Phenomena (Academic Press, San Diego, 1995).

[9] A. Lagendijk, B.A. van Tiggelen, Resonant Multiple Scattering of Light, Phys. Rep. 270, 143 (1996).

[10] D.S. Wiersma, P. Bartolini, Ad Lagendijk, R. Righini, Nature 390, 671 (1997).

[11] A.A. Chabanov, M. Stoytchev, A.Z. Genack, Nature 404, 850 (2000).

[12] M.I. Mishchenko, Astrophys. J. 411, 351 (1993).

[13] G. Labeyrie, F. De Tomasi, J-C Bernard, C.A. Müller, Ch. Miniatura, R. Kaiser, Phys. Rev. Lett. 83, 5266 (1999).

[14] G. Labeyrie, C.A. Muller, D.S. Wiersma, Ch. Miniatura, and R. Kaiser, J. Opt. B: Quantum Semiclass. Opt 2, 672 (2000).

[15] D.V. Kupriyanov, I.M. Sokolov, M.D. Havey, Optics Comm. 243, 165 (2004); D.V. Kupriyanov, N.V. Larionov, I.M. Sokolov, and M.D. Havey Optics and Spectroscopy 99 (3), 362 (2005).

[16] D.V. Kupriyanov, I.M. Sokolov, P. Kulatunga, C.I. Sukenik, M.D. Havey, Phys. Rev. A 67, 013814 (2003).

[17] J. Ishimaru and Yu. Kuga, J. Opt. Soc. Am. A1, 813 (1984).

[18] P.E. Wolf and G. Maret, Phys. Rev. Lett. 55, 2696 (1985).

[19] M.P. VanAlbada and A. Lagendijk, Phys. Rev. Lett. 55, 2692 (1985).

[20] Mark D. Havey and Dmitriy V. Kupriyanov, Physica
Scripta 72, C30,(2005).

[21] D.V. Kupriyanov, I.M. Sokolov, C.I. Sukenik, and M.D. Havey, Las. Phys. Lett. 3, 223 (2006).

[22] P.W. Anderson, Phys. Rev. 109, 1492 (1958).

[23] M.D. Lukin, Rev. Mod. Phys. 75, 457 (2003).

[24] E. Arimondo and G. Orriols, Nuovo Cimento Lett. 17, 333 (1976).

[25] H.R. Gray, R. M. Whitley, and C.R. Sroud, Opt. Lett. 3, 218 (1978).

[26] H.I. Yoo and J.H. Eberly, Phys. Rep. 118, 239 (1985).

[27] in Progress in Optics, ed. E. Wolf (Elsevier Science, Amsterdam, 1996), Vol. XXXV, p. 257).

[28] S.E. Harris, Phys. Today 50, 36 (1997).

[29] J.P. Marangos, J. Mod. Opt. 45, 471 (1998)

[30] R. Wynands and A. Nagel, Appl. Phys. B 68, 1 (1999) .

[31] L.V. Hau, S.E. Harris, Z. Dutton, C.H. Behroozi, Nature 397, 594 (1999).

[32] Danielle A. Braje, Vlatko Baic, Sunil Goda, G.Y. Yin, and S.E. Harris, Phys. Rev. Lett. 93, 183601 (2004).

[33] Chien Liu, Zachary Dutton, Cyrus H. Behroozi, and Lene Vestergaard Hau, Nature 409, 490 (2001).

[34] Danielle A. Braje, Vlatko Balic, G.Y. Yin, and S.E. Harris, Phys. Rev. A 68, 041801 (2003).

[35] T. Chanelire, D. N. Matsukevich, S. D. Jenkins, S.-Y. Lan, T. A. B. Kennedy, and A. Kuzmich, Nature 438, 833 (2005).

[36] M. D. Eisaman, A. Andr, F. Massou, M. Fleischhauer , A. S. Zibrov, and M. D. Lukin, Nature 438, 837 (2005).

[37] Min Yan, Edward G. Rickey, and Yifu Zhu, J. Opt. Soc. Am. B 18, 1057 (2001).

[38] Note that in warm atomic vapors, thermal atomic motion washes out the interferences. A.B. Matsko, I. Novikova, M.O. Scully, and G.R. Welch, Phys. Rev. Lett. 87, 133601 (2001).

[39] D.V. Datsyuk, I.M. Sokolov, JETP, 129 1-14 (2006). 